\title{
Functional characterization of two newly identified Human Endogenous Retrovirus coding envelope genes Sandra Blaise ${ }^{\dagger 2}$, Nathalie de Parseval ${ }^{\dagger 1}$ and Thierry Heidmann*1
}

\author{
Address: ${ }^{1}$ Unité des Rétrovirus Endogènes et Eléments Rétroïdes des Eucaryotes Supérieurs, UMR 8122 CNRS, Institut Gustave Roussy, 39 rue \\ Camille Desmoulins, 94805 Villejuif Cedex, France and ${ }^{2}$ Unité de Biologie des Rétrovirus, Département de Virologie, Institut Pasteur, 25 rue du \\ Dr Roux, 75724 Paris cedex 15, France \\ Email: Sandra Blaise - sblaise@pasteur.fr; Nathalie de Parseval - parseval@igr.fr; Thierry Heidmann* - heidmann@igr.fr \\ * Corresponding author †Equal contributors
}

Published: I 4 March 2005

Retrovirology 2005, 2:19 doi:10.1186/1742-4690-2-19

This article is available from: http://www.retrovirology.com/content/2/1/19

(C) 2005 Blaise et al; licensee BioMed Central Ltd.

This is an Open Access article distributed under the terms of the Creative Commons Attribution License (http://creativecommons.org/licenses/by/2.0), which permits unrestricted use, distribution, and reproduction in any medium, provided the original work is properly cited.
Received: 27 January 2005
Accepted: 14 March 2005

\begin{abstract}
A recent in silico search for coding sequences of retroviral origin present in the human genome has unraveled two new envelope genes that add to the 16 genes previously identified. A systematic search among the latter for a fusogenic activity had led to the identification of two bona fide genes, named syncytin-I and syncytin-2, most probably co-opted by primate genomes for a placental function related to the formation of the syncytiotrophoblast by cell-cell fusion. Here, we show that one of the newly identified envelope gene, named env $\mathrm{P}(\mathrm{b})$, is fusogenic in an ex vivo assay, but that its expression - as quantified by real-time RT-PCR on a large panel of human tissues - is ubiquitous, albeit with a rather low value in most tissues. Conversely, the second envelope gene, named envV, discloses a placenta-specific expression, but is not fusogenic in any of the cells tested. Altogether, these results suggest that at least one of these env genes may play a role in placentation, but most probably through a process different from that of the two previously identified syncytins.
\end{abstract}

\section{Findings}

Endogenous retroviral sequences represent approximately $8 \%$ of the human genome. These sequences (called HERVs for Human Endogenous Retroviruses) share strong similarities with present-day retroviruses, and are the proviral remnants of ancestral germ-line infections by active retroviruses, which have thereafter been transmitted in a Mendelian manner (reviewed in [1-3]). The 30,000 HERV elements have been grouped according to sequence homologies into more than 80 distinct families (each originating from the same founder element), based on a systematic listing of human repeats in the Repbase database [4]. Most of these elements are non-coding due to the accumulation of mutations, deletions, and/or truncations. A screening of the human genome for retroviral envelope genes with coding capacity, based on a specific envelope protein motif and on the HERV families described in Repbase, has revealed 16 fully coding envelope genes, transcribed in several healthy tissues $[5,6]$, among which two (syncytin-1 and syncytin-2) possess a fusogenic activity $[7,8]$. Using another approach, based on BLAST searches with various retroviral sequences as queries, a recent elegant study has analyzed the coding potential of human retroviral sequences and two additional fully coding envelope genes have emerged from this screen [9]. These two envelope genes do not belong to the HERV families listed in Repbase. The first one was designated "HERV-W/FRD-like" env, due to partial homology with syncytin-1 and syncytin-2, encoded by proviruses of the HERV-W and HERV-FRD families, respectively $[7,8]$. The second one was designated "ZFERV-like" env, due to its homology with the envelope protein encoded by a 


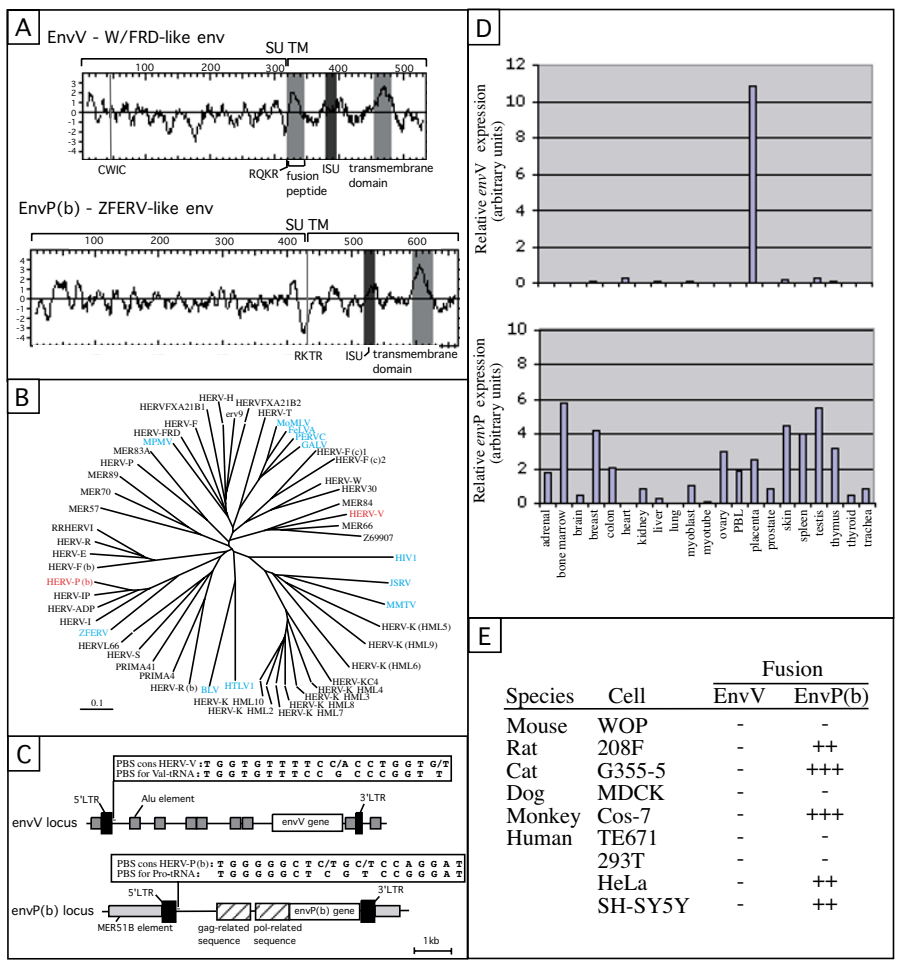

\section{Figure I}

A) Hydrophobicity profile and predicted features of the EnvV (formerly W/FRD-like env) and EnvP(b) (formerly ZFERV-like env) proteins. The SU (Surface Unit) and TM (TransMembrane) moieties of the envelopes are delineated, with the position of the putative proteolytic cleavage site (consensus, R/K-X-R/K-R) between the two subunits and the «CWLC » motif (consensus, $\mathrm{C}-\mathrm{X}-\mathrm{X}-\mathrm{C}$ ) indicated. The hydrophobic regions associated with the fusion peptide and the transmembrane region are shaded in light gray, and the putative immunosuppressive domain (ISU) in dark gray. B) Phylogenetic tree of retroviral envelopes and position of the newly identified genes. The tree is based on an alignment of approximately 180 amino acids corresponding to the extracellular and transmembrane domains of the TM subunit of envelope proteins. The protein alignment, phylogenetic tree and bootstrap analysis were performed with the ClustalW program (neighbour joining option). The tree was viewed by using the TreeView program. The scale bar indicates 10\% aa sequence difference. The phylogenetic tree determined by the parsimony method was congruent with the neighbour joining tree (data not shown). The two "new" $V$ and $P(b)$ env genes are represented in red, ERV env genes from other species and exogenous retroviruses in blue. The sequences used for the alignments were those of the consensus element of each family, or the coding env gene when present. The consensus sequences of the HERVK(HML-9), HERVFXA2 IBI and HERVFXA2 IB2 families, which are not listed in Repbase, were each inferred from the comparison of 3-6 sequences. Abbreviations: MoMLV, Moloney Murine Leukemia Virus; FeLVA, Feline Leukemia Virus strain A; PERVC, Pig Endogenous Retrovirus strain C; GALV, Gibbon Ape Leukemia Virus; MPMV, MazonPfizer Monkey Virus; MMTV, Mouse Mammary Tumor Virus; JSRV, Jaaksiekte Sheep Retrovirus; HTLV, Human T cell leukemia Virus; BLV, Bovine Leukemia Virus; HIV, Human Immunodeficiency Virus. C) Genomic organization of the envV and envP(b) loci. The envelope ORF (open box) with gag- and pol- related sequences (hatched boxes) and long terminal repeats (black boxes), Alu (dark gray boxes) and MER5IB (light gray boxes) retroelements are indicated. Consensus PBS sequences (obtained from two sequences for the HERV-V family and from four sequences for the HERV-P(b) family) are indicated above the corresponding provirus, together with the PBS for the $V$ al and Pro-tRNA, respectively. D) envV and envP(b) mRNA expression in a panel of 19 healthy human tissues, as determined by real-time quantitative RT-PCR. RNAs from human tissues were prepared as described in [6]. The reaction was performed using Sybr Green Master Mix (Applied Biosystems). PCR was developed using an ABI PRISM 7000 sequence detection system. Primer sequences (5'-3') were as follows: (CATGACTTTGGAAAAGGAGG) and (GCCAAAGAGGAAAAGTAAGAGT) for env); (CAAGATTGGGTCCCCTCAC) and (CCTATGGGGTCTTTCCCTC) for envP(b). The transcript levels were normalized relative to the amount of I8S mRNA (as determined with the primers and TaqMan probe from Applied Biosystems). Samples were assayed in duplicate. PBL, peripheral blood lymphocytes. E) Assay for fusogenicity of envV and envP(b). Xhol containing primer sequences (5'-3') were as follows: (ATCACCTCGAGACACTCCATCGAACCACTTCAT) and (ATCACCTCGAGGGCTGTTCTAGGATGGGTTATT) for env; (ATCACCTCGAGAGAAGAGAAACTTGAACCGTCC) and (ATCACCTCGAGGGGCTGATAGATGAATGGGTAT) for envP(b). The PCR products were cloned into the phCMV-G vector, opened with Xhol, and the constructs were verified by partial sequencing. Cell lines and fusion assays are as described in [12], except for the SH-SY5Y neuroblastoma cell line (ATCC number CRL-2266). 
provirus recently discovered in the zebrafish genome [10]. The sequences and predicted hydrophobic profiles of the two proteins (renamed here EnvV and EnvP(b) respectively, see below), disclose the characteristic signature of retroviral envelope proteins, with a putative proteolytic cleavage site between the SUrface (SU) and TransMembrane (TM) moieties, and a hydrophobic transmembrane domain within the TM subunit which permits its anchorage to the membrane (Figure 1A).

Since these genes belong to previously uncharacterized HERV families, we first analyzed their phylogenetic relationship with known HERV families and animal retroviruses. We generated a phylogenetic tree of endogenous and exogenous retroviruses based on the env gene, namely on the alignment of a conserved domain of the transmembrane (TM) subunit $[3,5]$. In this tree (Figure 1B), the "HERV-W/FRD-like" env gene is closely related to that of MER66, MER84 and Z69907 families. This gene seems to be part of a very degenerate proviral structure, with only the LTR being identifiable (see below and Figure 1C). As mentioned in [9], a highly homologous gene $(95.7 \%$ identity at the nucleotide level) encoding an envelope protein truncated due to a frameshift can be found $40 \mathrm{~kb}$ downstream. This cognate env gene is unambiguously part of a proviral structure, displaying just upstream of it the $1.6 \mathrm{~kb}$ open reading frame of a gag gene, followed by a pollike non coding region (data not shown. The flanking sequences of both proviruses are distinct. No other provirus or env gene belonging to this "family" can be found in the human genome by a BLAST search on the Ensembl database. Approximately $4 \mathrm{~kb}$ upstream of each of these two env genes, as expected, the RepeatMasker program that screens DNA sequences for interspersed repeats present in mammalian genomes http://www.repeatmas ker.org identifies 5' LTR sequences (or fragments of LTR sequences). 3' LTRs are also found just downstream of the envelope genes (see Figure 1B for the map of the fully coding env gene locus). The analysis of the PBS (Primer Binding Site) region located downstream of the two 5' LTRs of this family reveals a high degree of homology to the PBS for Val-tRNA (Figure 1C), so we propose to name this new family HERV-V.

The "ZFERV-like" env gene clusters, in the TM-based tree, with the "HERV-I superfamily", which indeed also includes the ZFERV env from zebrafish (see Figure 1B). As indicated in the retrosearch database http://www.retrose arch.dk, this envelope gene is part of an identifiable provirus (see Figure 1C). A BLAST query on the Ensembl database using the provirus sequence showed that this new HERV family contains three additional members. All four HERV elements, harbouring a proviral LTR-gag-pol-envLTR structure (although the only coding gene is the env gene described in [9]), are close to - but yet unambigu- ously distinct from - the HERV-IP family. The analysis of the PBS region of these four proviruses reveals a high degree of homology to the PBS for Pro-tRNA (see Figure 1C), so we propose to name this new family HERV-P(b) (since the HERV-P family already exists, [11]).

To determine whether these two genes could play a role in human placentation, we then characterized their expression pattern and fusogenic properties, as previously performed for the 16 coding envelope genes already identified $[6,8]$. To get insight into their expression profile, we used a Real-Time RT-PCR strategy as described in [6]. In this study, specific primers had been designed for Sybr Green amplification in such a way that only env genes with an open reading frame would be amplified among all the envelope genes of a given family, by positioning them within domains of maximal divergence between the coding and the non-coding copies. For the HERV-V coding envelope, the primer pair was designed in the 3' part of the gene, where the two env $\mathrm{V}$ genes are the most divergent ( $79 \%$ identity in the last $200 \mathrm{nt}$ ). An additional primer pair was also designed to monitore the expression of the truncated HERV-V env gene. To assess the specificity of each primer pair for the corresponding env gene, the PCR products obtained upon amplification of genomic DNA were cloned into a pGEM-T vector and 6 clones per amplicon were sequenced. In each case, the 6 sequences corresponded to the expected env gene. Analysis of the expression level of the coding envP(b) and $e n v \mathrm{~V}$ genes was achieved on a series of 19 healthy human tissues, and the results are represented in Figure 1D. The expression pattern of env $\mathrm{V}$ was found to be placenta-specific. Interestingly, the truncated envelope of the HERV-V family is highly expressed in the placenta as well, but poorly in other tissues (data not shown). EnvP(b) expression, on the other hand, was observed at a rather low level in almost all the tissues tested, without any specificity for the placenta.

Among the 16 coding env genes of the human genome tested in [8], only two, namely envW (syncytin-1) and envFRD (syncytin-2), had been found to be fusogenic in an $e x$ vivo assay. As these two env genes were highly and specifically expressed in the placenta, it was suggested that they are involved in a major physiological process within this organ, namely fusion of the cytotrophoblast cells to form the syncytiotrophoblast layer. The two newly identified env genes were therefore similarly tested. To do so, they were first cloned and introduced into a eukaryotic expression vector. The env $\mathrm{P}(\mathrm{b})$ gene was PCR-amplified from the DNA of BAC RP11-828K24 by using a proofreading DNA polymerase and running a 15-cycle PCR reaction, whereas the env $\mathrm{V}$ gene -not available as BAC DNA- was PCR amplified from the genomic DNA of a Caucasian individual using the Expand long template enzyme mix (Roche 
Applied Science). Both $e n v$ genes were then assayed for cell-cell fusion on a large panel of mammalian cells (known to express on the whole the receptors for all retroviral envelopes identified to date) using a transient transfection assay and two clones from each construct. As shown in Figure 1E, cell-cell fusion was observed in five out of nine cell lines tested for $e n v \mathrm{P}(\mathrm{b})$, and in none of them for $e n v \mathrm{~V}$. The truncated envelope protein member of the HERV-V family was also tested and, as expected, was not fusogenic (data not shown). In some respect, these results are surprising. Indeed, the putative protein encoded by env $\mathrm{P}(\mathrm{b})$ is fusogenic despite the absence of a canonical fusion peptide, i.e. of a hydrophobic region located at the N-terminus of the putative TM subunit, just downstream of the SU-TM cleavage site (see Figure 1A). Conversely, the env $\mathrm{V}$ gene product, notwithstanding its canonical sequence, is not fusogenic (at least in the panel of cells tested). To check that the lack of fusogenicity of the latter gene is not due to a fortuitous gene polymorphism of the $e n v \mathrm{~V}$ gene from the selected individual, we PCR-amplified, cloned and assayed the envV gene from two other individuals (for both the complete and the truncated $e n v \mathrm{~V}$ genes): no cell-cell fusion was observed either (data not shown). Finally, we identified and cloned the chimpanzee orthologous $e n v \mathrm{~V}$ gene (which is fully coding as well): neither did it display any fusogenic activity in our assay (data not shown).

In conclusion, the present analysis shows, rather paradoxically, that the envelope protein with fusogenic properties is not placenta-specific, whereas the one which is exclusively expressed in the placenta -a characteristic pattern of the two previously described fusogenic syncytin-1 and syncytin-2 gene products- is not fusogenic. In this respect, these results suggest that the two newly identified $e n v \mathrm{~V}$ and $e n v \mathrm{P}(\mathrm{b})$ genes are most probably not "syncytin-like" genes, sensu stricto. Additional experiments should now be devised (e.g. search for conservation among primates, search for Single Nucleotide Polymorphisms) to assess their role -if any- in human physiology.

\section{List of abbreviations}

HERV, human endogenous retrovirus; TM, transmembrane; LTR, Long Terminal Repeat; PBS, Primer Binding Site.

\section{Competing interests}

The author(s) declare that they have no competing interests.

\section{Authors' contributions}

SB carried out the cloning of the env genes and the cell-cell fusion assays.
NdP analyzed the sequences, constructed the phylogenetic tree, designed and carried out the Real-Time RT-PCR experiments, and drafted the manuscript.

TH conceived the study.

\section{Acknowledgements}

This work was supported by the CNRS and by grants from the Ligue Nationale contre le Cancer (Equipe Labellisée). We thank Christian Lavialle for critical reading of the manuscript.

\section{References}

I. Bannert N, Kurth R: Retroelements and the human genome: New perspectives on an old relation. Proceedings of the National Academy of Sciences of the United States of America 2004, I 3 Suppl 2: |4572-I4579.

2. Boeke JD, Stoye JP: Retrotransposons, endogenous retroviruses, and the evolution of retroelements. In Retroviruses Edited by: Coffin JM, Hughes SH and Varmus HE. New York, Cold Spring Harbor Laboratory Press; 1997:343-436.

3. de Parseval N, Heidmann T: Human endogenous retroviruses: from infectious elements to human genes. Cytogenetic and Genome Research in press.

4. Jurka J: Repbase update, a database an an electronic journal of repetitive elements. Trends in Genetics 2000, I 6:4 I 8-420.

5. Benit L, Dessen P, Heidmann T: Identification, phylogeny, and evolution of retroviral elements based on their envelope genes. Journal of Virology 2001, 75: I I709-II7I9.

6. de Parseval N, Lazar V, Casella JF, Benit L, Heidmann T: Survey of human genes of retroviral origin: identification and transcriptome of the genes with coding capacity for complete envelope proteins. J Virol 2003, 77:104|4-10422.

7. Blond JL, Lavillette D, Cheynet V, Bouton O, Oriol G, Chapel-Fernandes S, Mandrand B, Mallet F, Cosset FL: An envelope glycoprotein of the human endogenous retrovirus HERV-W is expressed in the human placenta and fuses cells expressing the type D mammalian retrovirus receptor. Journal of Virology 2000, 74:3321-3329.

8. Blaise S, de Parseval N, Bénit L, Heidmann T: Genomewide screening for fusogenic human endogenous retrovirus envelopes identifies syncytin 2 , a gene conserved on primate evolution. Proceedings of the National Academy of Sciences of the United States of America 2003, 100:13013-13018.

9. Villesen P, Aagaard L, Wiuf C, Pedersen FS: Identification of endogenous retroviral reading frames in the human genome. Retrovirology 2004, I:32.

10. Shen $\mathrm{CH}$, Steiner LA: Genome structure and thymic expression of an endogenous retrovirus in zebrafish. Journal of Virology 2004, 78:899-911.

II. Kröger B, Horak I: Isolation of novel human retrovirus-related sequences by hybridization to synthetic oligonucleotides complementary to the tRNA Pro primer-binding site. Journal of Virology 1987, 6 I:2071-2075.

12. Dupressoir A, Marceau G, Vernochet C, Benit L, Kanellopoulos C, Sapin V, Heidmann T: Syncytin-A and syncytin-B, two fusogenic placenta-specific murine envelope genes of retroviral origin conserved in Muridae. Proc Natl Acad Sci U S A 2005, 1 02:725-30. Epub 2005 Jan I I.. 